\title{
A Note on Stokes Approximations to Leray Solutions of the Incompressible Navier-Stokes Equations in $\mathbb{R}^{n}$
}

\author{
${\text { Joyce C. } \text { Rigelo }^{1}(\mathbb{D} \text {, Janaína P. Zingano }}^{2}\left(\mathbb{D}\right.$ and Paulo R. Zingano ${ }^{2, *(\mathbb{D})}$ \\ 1 Master AI, Inc., 10036 Milla Circle, Austin, TX 78748, USA; joycerigelo@gmail.com \\ 2 Departamento de Matemática Pura e Aplicada, Universidade Federal do Rio Grande do Sul, \\ Porto Alegre 91509, RS, Brazil; janaina.zingano@ufrgs.br \\ * Correspondence: paulo.zingano@ufrgs.br
}

check for

updates

Citation: Rigelo, J.C.; Zingano, J.P.; Zingano, P.R. A Note on Stokes Approximations to Leray Solutions of the Incompressible Navier-Stokes Equations in $\mathbb{R}^{n}$. Fluids 2021, 6, 340 . https: / / doi.org/10.3390/ fluids 6100340

Academic Editors: Ashwin Vaidya and Mehrdad Massoudi

Received: 29 July 2021

Accepted: 24 September 2021

Published: 27 September 2021

Publisher's Note: MDPI stays neutral with regard to jurisdictional claims in published maps and institutional affiliations.

Copyright: (c) 2021 by the authors. Licensee MDPI, Basel, Switzerland. This article is an open access article distributed under the terms and conditions of the Creative Commons Attribution (CC BY) license (https:/ / creativecommons.org/licenses/by/ $4.0 /)$.

\begin{abstract}
In the early 1980s it was well established that Leray solutions of the unforced Navier-Stokes equations in $\mathbb{R}^{n}$ decay in energy norm for large $t$. With the works of T. Miyakawa, M. Schonbek and others it is now known that the energy decay rate cannot in general be any faster than $t^{-(n+2) / 4}$ and is typically much slower. In contrast, we show in this note that, given an arbitrary Leray solution $\boldsymbol{u}(\cdot, t)$, the difference of any two Stokes approximations to the Navier-Stokes flow $\boldsymbol{u}(\cdot, t)$ will always decay at least as fast as $t^{-(n+2) / 4}$, no matter how slow the decay of $\|\boldsymbol{u}(\cdot, t)\|_{L^{2}\left(\mathbb{R}^{n}\right)}$ might be.
\end{abstract}

Keywords: Navier-Stokes equations; Stokes flows; Leray solutions; large time behavior

AMS Mathematics Subject Classification: 35Q30; 76D07 (primary); 76D05 (secondary)

\section{Introduction}

In this note, we derive an interesting new property regarding the large time behavior of Stokes flows approximating Leray solutions (as constructed by J. Leray in [1]) of the incompressible Navier-Stokes equations

$$
\begin{aligned}
\boldsymbol{u}_{t}+\boldsymbol{u} \cdot \nabla \boldsymbol{u}+\nabla p & =v \Delta \boldsymbol{u}, \quad \nabla \cdot \boldsymbol{u}(\cdot, t)=0, \\
\boldsymbol{u}(\cdot, 0) & =\boldsymbol{u}_{0} \in L_{\sigma}^{2}\left(\mathbb{R}^{n}\right),
\end{aligned}
$$

in dimension $2 \leq n \leq 4$, where $v>0$ is constant and $L_{\sigma}^{2}\left(\mathbb{R}^{n}\right)$ denotes the space of functions $\mathbf{u}=\left(\mathbf{u}_{1}, \ldots, \mathbf{u}_{n}\right) \in L^{2}\left(\mathbb{R}^{n}\right)$ with $\nabla \cdot \mathbf{u}=0$ in the distributional sense. Leray solutions to (1),(2) are mappings $\boldsymbol{u}(\cdot, t) \in L^{\infty}\left((0, \infty), L_{\sigma}^{2}\left(\mathbb{R}^{n}\right)\right) \cap L^{2}\left((0, \infty), \dot{H}^{1}\left(\mathbb{R}^{n}\right)\right)$ that are weakly continuous in $L^{2}\left(\mathbb{R}^{n}\right)$ for all $t \geq 0$ and satisfy the Equation $(1)$ in $\mathbb{R}^{n} \times(0, \infty)$ as distributions. Moreover, they satisfy the energy estimate [1-6]

$$
\|\boldsymbol{u}(\cdot, t)\|_{L^{2}\left(\mathbb{R}^{n}\right)}^{2}+2 v \int_{0}^{t}\|D \boldsymbol{u}(\cdot, s)\|_{L^{2}\left(\mathbb{R}^{n}\right)}^{2} d s \leq\left\|\boldsymbol{u}_{0}\right\|_{L^{2}\left(\mathbb{R}^{n}\right)}^{2}
$$

(for all $t \geq 0$ ), so that, in particular, $\left\|\boldsymbol{u}(\cdot, t)-\boldsymbol{u}_{0}\right\|_{L^{2}\left(\mathbb{R}^{n}\right)} \rightarrow 0$ as $t \searrow 0$. For $n \geq 3$, their uniqueness and exact regularity properties are still an open problem, but it is known that at the very least they must be smooth for large $t$ : for some $t_{*} \geq 0$ (depending on the solution) we have $u \in C^{\infty}\left(\mathbb{R}^{n} \times\left(t_{*}, \infty\right)\right)$, and, for each $m \geq 0$,

$$
\boldsymbol{u}(\cdot, t) \in C\left(\left(t_{*}, \infty\right), H^{m}\left(\mathbb{R}^{n}\right)\right),
$$

as shown by Leray ([1], p. 246). Actually, we have

and 


$$
t_{*} \leq 0.002728 v^{-3}\left\|\boldsymbol{u}_{0}\right\|_{L^{2}\left(\mathbb{R}^{4}\right)}^{2} \quad(\text { if } n=4)
$$

(see [7],THEOREM A), with $t_{*}=0$ if $n=2$. For more on solution properties, see e.g., [1-6,8-11]. Here, we are particularly interested in the behavior for $t \gg 1$ : it is now well known that, for every $m \geq 0$,

$$
\lim _{t \rightarrow \infty} t^{m / 2}\left\|D^{m} \boldsymbol{u}(\cdot, t)\right\|_{L^{2}\left(\mathbb{R}^{n}\right)}=0
$$

(see e.g., [11-21]), where $\left\|D^{m} \boldsymbol{u}(\cdot, t)\right\|_{L^{2}\left(\mathbb{R}^{n}\right)}$ denotes the norm of $\boldsymbol{u}(\cdot, t)$ in $\dot{H}^{m}\left(\mathbb{R}^{n}\right)$, that is,

$$
\|\boldsymbol{u}(\cdot, t)\|_{L^{2}\left(\mathbb{R}^{n}\right)}^{2}=\left\|u_{1}(\cdot, t)\right\|_{L^{2}\left(\mathbb{R}^{n}\right)}^{2}+\ldots+\left\|u_{n}(\cdot, t)\right\|_{L^{2}\left(\mathbb{R}^{n}\right)}^{2}
$$

if $m=0$ and

$$
\left\|D^{m} \boldsymbol{u}(\cdot, t)\right\|_{L^{2}\left(\mathbb{R}^{n}\right)}^{2}=\sum_{i=1}^{n} \sum_{j_{1}=1}^{n} \cdots \sum_{j_{m}=1}^{n} \int_{\mathbb{R}^{n}}\left|D_{j_{1}} \cdots D_{j_{m}} u_{i}(x, t)\right|^{2} d x
$$

if $m \geq 1$, where $D_{j}=\partial / \partial x_{j}$. For arbitrary initial values $\boldsymbol{u}_{0} \in L_{\sigma}^{2}\left(\mathbb{R}^{n}\right)$, the result (7) is all that can be obtained, but stronger additional assumptions may give that, for some $\alpha>0$, we actually have

$$
\left\|D^{m} \boldsymbol{u}(\cdot, t)\right\|_{L^{2}\left(\mathbb{R}^{n}\right)}=O\left(t^{-\alpha-m / 2}\right)
$$

as $t \rightarrow \infty$, with the generic limitation $\alpha \leq(n+2) / 4$, see $[8,22,23]$. (For the exceptional case of faster decaying solutions, see $[8,22,24,25]$.) Another point of interest is the large time behavior of the associated linear Stokes flows. In the case of (1), (2) these are given by solutions $v(\cdot, t) \in L^{\infty}\left(\left[t_{0}, \infty\right), L_{\sigma}^{2}\left(\mathbb{R}^{n}\right)\right)$ of the linear heat flow problems

$$
\begin{gathered}
\boldsymbol{v}_{t}=v \Delta \boldsymbol{v}, \quad t>t_{0}, \\
\boldsymbol{v}\left(\cdot, t_{0}\right)=\boldsymbol{u}\left(\cdot, t_{0}\right),
\end{gathered}
$$

for some given $t_{0} \geq 0$ (arbitrary). The solution is given by $\boldsymbol{v}(\cdot, t)=e^{v \Delta\left(t-t_{0}\right)} \boldsymbol{u}\left(\cdot, t_{0}\right)$, where $e^{v \Delta \tau}, \tau \geq 0$, is the heat semigroup. If $\alpha<(n+2) / 4$, the error $\left\|D^{m}(\boldsymbol{u}-\boldsymbol{v})(\cdot, t)\right\|_{L^{2}\left(\mathbb{R}^{n}\right)}$ decays faster than the rate (10), so that the Stokes solutions (11), (12) give a useful approximation to the more complex Navier-Stokes flow $\boldsymbol{u}(\cdot, t)$ defined by the Equation (1).

Our contribution in this note is to point out that, for arbitrary Navier-Stokes flows (i.e., for arbitrary initial values $\left.\boldsymbol{u}_{0} \in L_{\sigma}^{2}\left(\mathbb{R}^{n}\right)\right)$, two distinct Stokes approximations $\boldsymbol{v}(\cdot, t), \tilde{\boldsymbol{v}}(\cdot, t)$ to $\boldsymbol{u}(\cdot, t)$ eventually become very closely similar in that we always have

$$
\left\|D^{m}[\boldsymbol{v}(\cdot, t)-\tilde{\boldsymbol{v}}(\cdot, t)]\right\|_{L^{2}\left(\mathbb{R}^{n}\right)}=O\left(t^{-(n+2) / 4-m / 2}\right)
$$

for large $t$. The precise statement reads as follows:

Theorem 1. Given $2 \leq n \leq 4$ and $\boldsymbol{u}_{0} \in L_{\sigma}^{2}\left(\mathbb{R}^{n}\right)$, let $\boldsymbol{u}(\cdot, t) \in L^{\infty}\left((0, \infty), L_{\sigma}^{2}\left(\mathbb{R}^{n}\right)\right) \cap L^{2}((0, \infty)$, $\left.\dot{H}^{1}\left(\mathbb{R}^{n}\right)\right)$ be any Leray solution to the Navier-Stokes equations (1). Then, for any $0 \leq t_{0}<\tilde{t}_{0}$, we have

$$
\|\boldsymbol{v}(\cdot, t)-\tilde{\boldsymbol{v}}(\cdot, t)\|_{L^{2}\left(\mathbb{R}^{n}\right)} \leq K v^{-(n+2) / 4}\|\boldsymbol{u}(\cdot, 0)\|_{L^{2}\left(\mathbb{R}^{n}\right)}^{2}\left(\tilde{t}_{0}-t_{0}\right)\left(t-\tilde{t}_{0}\right)^{-(n+2) / 4}
$$

for all $t>\tilde{t}_{0}$, where $\boldsymbol{v}(\cdot, t)=e^{v \Delta\left(t-t_{0}\right)} \boldsymbol{u}\left(\cdot, t_{0}\right)$ and $\tilde{\boldsymbol{v}}(\cdot, t)=e^{v \Delta\left(t-\tilde{t}_{0}\right)} \boldsymbol{u}\left(\cdot, \tilde{t}_{0}\right)$ are the corresponding Stokes flows associated with the time instants $t_{0}$ and $\tilde{t}_{0}$, respectively, and $K=(4 \pi)^{-n / 4} / \sqrt{e}$. Moreover, for any $m \geq 1$, we have

$$
\left\|D^{m}[\boldsymbol{v}(\cdot, t)-\tilde{\boldsymbol{v}}(\cdot, t)]\right\|_{L^{2}\left(\mathbb{R}^{n}\right)} \leq K(m, n) v^{-(n+2) / 4-m / 2}\left\|\boldsymbol{u}_{0}\right\|_{L^{2}\left(\mathbb{R}^{n}\right)}^{2}\left(\tilde{t}_{0}-t_{0}\right)\left(t-\tilde{t}_{0}\right)^{-(n+2) / 4-m / 2}
$$


for all $t>\tilde{t}_{0}$, where the constant $K(m, n)$ depends only on $m, n$ (and not on $t_{0}, \tilde{t}_{0}, v, \boldsymbol{u}_{0}$ or the solution $\boldsymbol{u}(\cdot, t))$.

Remark 1. In dimension $n \geq 3$ it is not known whether Leray's construction gives all (weak) solutions in the class $X=\left\{\boldsymbol{u}(\cdot, t) \in L^{\infty}\left((0, \infty), L_{\sigma}^{2}\left(\mathbb{R}^{n}\right)\right) \cap L^{2}\left((0, \infty), \dot{H}_{\sigma}^{1}\left(\mathbb{R}^{n}\right)\right)\right.$ : (3) holds for all $t>0\}$, the so-called Leray-Hopf solutions. In case it does not, it would be interesting to know if THEOREM A remains valid for all Leray-Hopf solutions as well.

From (14), (15) and standard Sobolev imbeddings we obtain the following corollary regarding supnorm estimates.

Theorem 2. Given $2 \leq n \leq 4$ and $\boldsymbol{u}_{0} \in L_{\sigma}^{2}\left(\mathbb{R}^{n}\right)$, let $\boldsymbol{u}(\cdot, t) \in L^{\infty}\left((0, \infty), L_{\sigma}^{2}\left(\mathbb{R}^{n}\right)\right) \cap L^{2}((0, \infty)$, $\left.\dot{H}^{1}\left(\mathbb{R}^{n}\right)\right)$ be any Leray solution to the Navier-Stokes equations (1). Then, for any $0 \leq t_{0}<\tilde{t}_{0}$, we have

$\left\|D^{m}[\boldsymbol{v}(\cdot, t)-\tilde{\boldsymbol{v}}(\cdot, t)]\right\|_{L^{\infty}\left(\mathbb{R}^{n}\right)} \leq G(m, n) v^{-(n+2) / 4-m / 2-n / 4}\|\boldsymbol{u}(\cdot, 0)\|_{L^{2}\left(\mathbb{R}^{n}\right)}^{2}\left(\tilde{t}_{0}-t_{0}\right)\left(t-\tilde{t}_{0}\right)^{-(n+2) / 4-m / 2-n / 4}$

for all $t>\tilde{t}_{0}$ and every $m \geq 0$, where $\boldsymbol{v}(\cdot, t)=e^{v \Delta\left(t-t_{0}\right)} \boldsymbol{u}\left(\cdot, t_{0}\right)$ and $\tilde{\boldsymbol{v}}(\cdot, t)=e^{v \Delta\left(t-\tilde{t}_{0}\right)} \boldsymbol{u}\left(\cdot, \tilde{t}_{0}\right)$, and where $G(m, n)>0$ is some constant that depends only on $(m, n)$.

Remark 2. Earlier versions of (14) and (16) for $m=0$ were given in [26,27], but, as the results and analysis there were neither as sharp nor as complete as in the present discussion, they have now become obsolete.

The proof of Theorem 1 is developed in the next section, along with some necessary mathematical preliminaries. We end the discussion with some brief considerations in Section 3.

\section{Proof of Theorem 1}

We first recall Leray's construction [1], as it will be needed in the proof of Theorem 1 if $n \geq 3$. (If $n=2$, the proof can be done directly from (1) by easily adapting the argument below.) For the construction of his solutions, Leray used an ingenious regularization procedure which we now review. Taking (any) $G \in C_{0}^{\infty}\left(\mathbb{R}^{n}\right)$ nonnegative with $\int_{\mathbb{R}^{n}} G(x) d x=1$ and setting $\bar{u}_{0, \delta}(\cdot) \in C^{\infty}\left(\mathbb{R}^{n}\right)$ by convolving $u_{0}(\cdot)$ with $G_{\delta}(x)=\delta^{-n} G(x / \delta), \delta>0$, one defines $\boldsymbol{u}_{\delta^{\prime}}, p_{\delta} \in C^{\infty}\left(\mathbb{R}^{n} \times[0, \infty)\right)$ as the (unique, globally defined) classical $L^{2}$ solutions of the regularized equations

$$
\begin{gathered}
\frac{\partial}{\partial t} \boldsymbol{u}_{\delta}+\overline{\boldsymbol{u}}_{\delta}(\cdot, t) \cdot \nabla \boldsymbol{u}_{\delta}+\nabla p_{\delta}=\Delta \boldsymbol{u}_{\delta^{\prime}} \quad \nabla \cdot \boldsymbol{u}_{\delta}(\cdot, t)=0, \\
\boldsymbol{u}_{\delta}(\cdot, 0)=\overline{\boldsymbol{u}}_{0, \delta}:=G_{\delta} * \boldsymbol{u}_{0} \in \bigcap_{m=1}^{\infty} H^{m}\left(\mathbb{R}^{n}\right),
\end{gathered}
$$

where $\overline{\boldsymbol{u}}_{\delta}(\cdot, t):=G_{\delta} * \boldsymbol{u}_{\delta}(\cdot, t)$. As shown by Leray, there is some sequence $\delta^{\prime} \rightarrow 0$ for which we have the weak convergence

$$
\boldsymbol{u}_{\delta^{\prime}}(\cdot, t) \rightarrow \boldsymbol{u}(\cdot, t) \quad \text { as } \delta^{\prime} \rightarrow 0, \quad \forall t \geq 0,
$$

that is, $u_{\delta^{\prime}}(\cdot, t) \rightarrow \boldsymbol{u}(\cdot, t)$ weakly in $L^{2}\left(\mathbb{R}^{n}\right)$, for every $t \geq 0$ (see [1], p. 237). This gives $\boldsymbol{u}(\cdot, t) \in L^{\infty}\left((0, \infty), L_{\sigma}^{2}\left(\mathbb{R}^{n}\right)\right) \cap L^{2}\left((0, \infty), \dot{H}^{1}\left(\mathbb{R}^{n}\right)\right) \cap C_{w}^{0}\left([0, \infty), L^{2}\left(\mathbb{R}^{n}\right)\right)$, with $\boldsymbol{u}(\cdot, t)$ continuous in $L^{2}$ at $t=0$ and solving the Navier-Stokes Equations (1) in distributional sense. Moreover, the energy inequality (3) is satisfied for all $t \geq 0$, so that, in particular,

$$
\int_{0}^{\infty}\|D \boldsymbol{u}(\cdot, t)\|_{L^{2}\left(\mathbb{R}^{n}\right)}^{2} d t \leq \frac{1}{2 v}\left\|\boldsymbol{u}_{0}\right\|_{L^{2}\left(\mathbb{R}^{n}\right)}^{2} .
$$


A similar estimate for the regularized solutions $\boldsymbol{u}_{\delta}(\cdot, t)$ is also valid, since we have, from (17), (18) above, that

$$
\left\|\boldsymbol{u}_{\delta}(\cdot, t)\right\|_{L^{2}\left(\mathbb{R}^{n}\right)}^{2}+2 v \int_{0}^{t}\left\|D \boldsymbol{u}_{\delta}(\cdot, s)\right\|_{L^{2}\left(\mathbb{R}^{n}\right)}^{2} d s \leq\left\|\boldsymbol{u}_{0}\right\|_{L^{2}\left(\mathbb{R}^{n}\right)}^{2}
$$

for all $t>0$ (and $\delta>0$ arbitrary). Another property shown in [1] is that $u \in C^{\infty}\left(\mathbb{R}^{n} \times\left(t_{*}, \infty\right)\right)$ for some $t_{*} \geq 0$, with $D^{m} \boldsymbol{u}(\cdot, t) \in C\left(\left(t_{*}, \infty\right), L^{2}\left(\mathbb{R}^{n}\right)\right)$ for each $m \geq 1$, cf. (4). The following result considers the Helmholtz projection of $-\boldsymbol{u}(\cdot, t) \cdot \nabla \boldsymbol{u}(\cdot, t)$ into $L_{\sigma}^{2}\left(\mathbb{R}^{n}\right)$, that is, the divergence-free field $Q(\cdot, t) \in L_{\sigma}^{2}\left(\mathbb{R}^{n}\right)$ given by

$$
\boldsymbol{Q}(\cdot, t):=-\boldsymbol{u}(\cdot, t) \cdot \nabla \boldsymbol{u}(\cdot, t)-\nabla p(\cdot, t), \quad \text { a.e. } t>0
$$

Of similar interest is the quantity $\boldsymbol{Q}_{\delta}(\cdot, t):=-\overline{\boldsymbol{u}}_{\delta}(\cdot, t) \cdot \nabla \boldsymbol{u}_{\delta}(\cdot, t)-\nabla p_{\delta}(\cdot, t)$, which will be important in Theorem 4 below.

Theorem 3. For almost every $s>0$ (and every $s \geq t_{*}$, with $t_{*}$ given in (4) above), one has

$$
\begin{aligned}
& \left\|e^{v \Delta(t-s)} \boldsymbol{Q}(\cdot, s)\right\|_{L^{2}\left(\mathbb{R}^{n}\right)} \leq K_{1}(n) v^{-n / 4}(t-s)^{-n / 4}\|\boldsymbol{u}(\cdot, s)\|_{L^{2}\left(\mathbb{R}^{n}\right)}\|D \boldsymbol{u}(\cdot, s)\|_{L^{2}\left(\mathbb{R}^{n}\right)} \\
& \qquad \quad\left\|e^{v \Delta(t-s)} \boldsymbol{Q}(\cdot, s)\right\|_{L^{2}\left(\mathbb{R}^{n}\right)} \leq K_{2}(n) v^{-(n+2) / 4}(t-s)^{-(n+2) / 4}\|\boldsymbol{u}(\cdot, s)\|_{L^{2}\left(\mathbb{R}^{n}\right)}^{2}
\end{aligned}
$$

for all $t>s$, where $K_{1}(n)=(8 \pi)^{-n / 4}$ and $K_{2}(n)=(4 \pi)^{-n / 4} / \sqrt{e}$.

Proof. This is shown in [14], p. 236, using the Fourier transform. Here we give an alternative, direct argument in physical space: Let $\mathbb{P}$ be the Helmholtz projection. Since, by definition, $\mathbb{P}$ is an orthogonal projection in the Hilbert space of vector fields in $L^{2}$, we have $\|\mathbb{P} f\|_{L^{2}} \leq\|f\|_{L^{2}}$ for any vector field in $L^{2}$. Hence we have $\left\|e^{v \Delta(t-s)} Q(\cdot, s)\right\|_{L^{2}}=$ $\left\|e^{v \Delta(t-s)} \mathbb{P}[\boldsymbol{u}(\cdot, s) \cdot \nabla \boldsymbol{u}(\cdot, s)]\right\|_{L^{2}}=\left\|\mathbb{P}\left[e^{v \Delta(t-s)}(\boldsymbol{u}(\cdot, s) \cdot \nabla \boldsymbol{u}(\cdot, s))\right]\right\|_{L^{2}} \leq \| e^{v \Delta(t-s)}(\boldsymbol{u}(\cdot, s)$ $\cdot \nabla \boldsymbol{u}(\cdot, s))\left\|_{L^{2}} \leq\right\| \Gamma(t-s)\left\|_{L^{2}}\right\| \boldsymbol{u}(\cdot, s) \cdot \nabla \boldsymbol{u}(\cdot, s) \|_{L^{1}}$, where $\Gamma$ denotes the heat kernel, so that $\left\|e^{v \Delta(t-s)} \boldsymbol{Q}(\cdot, s)\right\|_{L^{2}} \leq\|\Gamma(t-s)\|_{L^{2}}\|\boldsymbol{u}(\cdot, s)\|_{L^{2}}\|\nabla \boldsymbol{u}(\cdot, s)\|_{L^{2}}$. This is (23). Similarly, $\left\|e^{v \Delta(t-s)} \boldsymbol{Q}(\cdot, s)\right\|_{L^{2}} \leq \sum_{j=1}^{n}\left\|\Gamma(t-s) * D_{j}\left[u_{j}(\cdot, s) \boldsymbol{u}(\cdot, s)\right]\right\|_{L^{2}}=\sum_{j=1}^{n} \| D_{j} \Gamma(t-s) *$ $\left[u_{j}(\cdot, s) \boldsymbol{u}(\cdot, s)\right]\left\|_{L^{2}} \leq \sum_{j=1}^{n}\right\| D_{j} \Gamma(t-s)\left\|_{L^{2}}\right\| u_{j}(\cdot, s) \boldsymbol{u}(\cdot, s)\left\|_{L^{1}} \leq \sum_{j=1}^{n}\right\| D_{j} \Gamma(t-$ s) $\left\|_{L^{2}}\right\| u_{j}(\cdot, s)\left\|_{L^{2}}\right\| \boldsymbol{u}(\cdot, s) \|_{L^{2}}$, which gives (24), as claimed.

In a completely similar way, considering the solutions of the regularized NavierStokes Equations (17) and (18), one obtains

$$
\begin{aligned}
& \left\|e^{v \Delta(t-s)} \boldsymbol{Q}_{\delta}(\cdot, s)\right\|_{L^{2}\left(\mathbb{R}^{n}\right)} \leq K_{1}(n) v^{-n / 4}(t-s)^{-n / 4}\left\|\boldsymbol{u}_{\delta}(\cdot, s)\right\|_{L^{2}\left(\mathbb{R}^{n}\right)}\left\|D \boldsymbol{u}_{\delta}(\cdot, s)\right\|_{L^{2}\left(\mathbb{R}^{n}\right)} \\
& \quad \text { and }
\end{aligned}
$$

$$
\left\|e^{v \Delta(t-s)} \boldsymbol{Q}_{\delta}(\cdot, s)\right\|_{L^{2}\left(\mathbb{R}^{n}\right)} \leq K_{2}(n) v^{-(n+2) / 4}(t-s)^{-(n+2) / 4}\left\|\boldsymbol{u}_{\delta}(\cdot, s)\right\|_{L^{2}\left(\mathbb{R}^{n}\right)}^{2}
$$

for all $t>s>0$, where the constants $K_{1}(n), K_{2}(n)$ are given in Theorem 3 and $\boldsymbol{Q}_{\delta}(\cdot, s)=-\overline{\boldsymbol{u}}_{\delta}(\cdot, s) \cdot \nabla \boldsymbol{u}_{\delta}(\cdot, s)-\nabla p_{\delta}(\cdot, s)$.

Theorem 4. Let $\boldsymbol{u}(\cdot, t), t>0$, be any particular Leray solution to (1). Given any pair of starting times $\tilde{t}_{0}>t_{0} \geq 0$, one has

$$
\|\boldsymbol{v}(\cdot, t)-\tilde{\boldsymbol{v}}(\cdot, t)\|_{L^{2}\left(\mathbb{R}^{n}\right)} \leq K_{2}(n) v^{-(n+2) / 4}\|\boldsymbol{u}(\cdot, 0)\|_{L^{2}\left(\mathbb{R}^{n}\right)}^{2}\left(\tilde{t}_{0}-t_{0}\right)\left(t-\tilde{t}_{0}\right)^{-(n+2) / 4}
$$

for all $t>\tilde{t}_{0}$, where $\boldsymbol{v}(\cdot, t)=e^{v \Delta\left(t-t_{0}\right)} \boldsymbol{u}\left(\cdot, t_{0}\right), \tilde{\boldsymbol{v}}(\cdot, t)=e^{v \Delta\left(t-\tilde{t}_{0}\right)} \boldsymbol{u}\left(\cdot, \tilde{t}_{0}\right)$ are the corresponding Stokes flows associated with $t_{0}, \tilde{t}_{0}$, respectively, and $K_{2}(n)$ is given in Theorem 3 above, that is, $K_{2}(n)=(4 \pi)^{-n / 4} / \sqrt{e}$. 
Proof. The following argument combines the Leray's construction reviewed above with the usual strategy of handling nonlinear terms as a Duhamel-type correction. We begin by writing $\boldsymbol{v}(\cdot, t)=e^{v \Delta\left(t-t_{0}\right)}\left[\boldsymbol{u}\left(\cdot, t_{0}\right)-\boldsymbol{u}_{\delta}\left(\cdot, t_{0}\right)\right]+e^{v \Delta\left(t-t_{0}\right)} \boldsymbol{u}_{\delta}\left(\cdot, t_{0}\right), t>t_{0}$, with $\boldsymbol{u}_{\delta}(\cdot, t)$ given in (17), (18), $\delta>0$. Because

$$
\boldsymbol{u}_{\delta}\left(\cdot, t_{0}\right)=e^{v \Delta t_{0}} \overline{\boldsymbol{u}}_{0, \delta}+\int_{0}^{t_{0}} e^{v \Delta\left(t_{0}-s\right)} \boldsymbol{Q}_{\delta}(\cdot, s) d s,
$$

where $\overline{\boldsymbol{u}}_{0, \delta}=G_{\delta} * \boldsymbol{u}_{0}$ and $\boldsymbol{Q}_{\delta}(\cdot, s)=-\overline{\boldsymbol{u}}_{\delta}(\cdot, s) \cdot \nabla \boldsymbol{u}_{\delta}(\cdot, s)-\nabla p_{\delta}(\cdot, s)$, we get

$$
\boldsymbol{v}(\cdot, t)=e^{v \Delta\left(t-t_{0}\right)}\left[\boldsymbol{u}\left(\cdot, t_{0}\right)-\boldsymbol{u}_{\delta}\left(\cdot, t_{0}\right)\right]+e^{v \Delta t} \overline{\boldsymbol{u}}_{0, \delta}+\int_{0}^{t_{0}} e^{v \Delta(t-s)} \boldsymbol{Q}_{\delta}(\cdot, s) d s,
$$

for $t>t_{0}$. A similar expression holds for $\tilde{\boldsymbol{v}}(\cdot, t)$ as well, giving

$$
\tilde{\boldsymbol{v}}(\cdot, t)-\boldsymbol{v}(\cdot, t)=e^{v \Delta\left(t-\tilde{t}_{0}\right)}\left[\boldsymbol{u}\left(\cdot, \tilde{t}_{0}\right)-\boldsymbol{u}_{\delta}\left(\cdot, \tilde{t}_{0}\right)\right]-e^{v \Delta\left(t-t_{0}\right)}\left[\boldsymbol{u}\left(\cdot, t_{0}\right)-\boldsymbol{u}_{\delta}\left(\cdot, t_{0}\right)\right]+\int_{t_{0}}^{\tilde{t}_{0}} e^{v \Delta(t-s)} \boldsymbol{Q}_{\delta}(\cdot, s) d s
$$

for $t>\tilde{t}_{0}$. Therefore, given any $\mathbb{K} \subset \mathbb{R}^{n}$ compact, we get, for each $t>\tilde{t}_{0}, \delta>0$ :

$$
\begin{aligned}
\|\tilde{\boldsymbol{v}}(\cdot, t)-\boldsymbol{v}(\cdot, t)\|_{L^{2}(\mathbb{K})} & \leq J_{\delta}(t)+\int_{t_{0}}^{\tilde{t}_{0}}\left\|e^{v \Delta(t-s)} Q_{\delta}(\cdot, s)\right\|_{L^{2}(\mathbb{K})} d s \\
& \leq J_{\delta}(t)+K_{2}(n) v^{-(n+2) / 4} \int_{t_{0}}^{\tilde{t}_{0}}(t-s)^{-(n+2) / 4}\left\|\boldsymbol{u}_{\delta}(\cdot, s)\right\|_{L^{2}\left(\mathbb{R}^{n}\right)}^{2} d s \\
& \leq J_{\delta}(t)+K_{2}(n) v^{-(n+2) / 4}\left\|\boldsymbol{u}_{0}\right\|_{L^{2}\left(\mathbb{R}^{n}\right)}^{2}\left(\tilde{t}_{0}-t_{0}\right)\left(t-\tilde{t}_{0}\right)^{-(n+2) / 4}
\end{aligned}
$$

by (21) and (26), where $K_{2}(n)=(4 \pi)^{-n / 4} / \sqrt{e}$ and

$$
J_{\delta}(t)=\left\|e^{v \Delta\left(t-\tilde{t}_{0}\right)}\left[\boldsymbol{u}\left(\cdot, \tilde{t}_{0}\right)-\boldsymbol{u}_{\delta}\left(\cdot, \tilde{t}_{0}\right)\right]\right\|_{L^{2}(\mathbb{K})}+\left\|e^{v \Delta\left(t-t_{0}\right)}\left[\boldsymbol{u}\left(\cdot, t_{0}\right)-\boldsymbol{u}_{\delta}\left(\cdot, t_{0}\right)\right]\right\|_{L^{2}(\mathbb{K})} .
$$

Taking $\delta=\delta^{\prime} \rightarrow 0$ according to (19), we get $J_{\delta}(t) \rightarrow 0$, since, by Lebesgue's Dominated Convergence Theorem and (19), we have, for any $\sigma, \tau>0:\left\|e^{v \Delta \tau}\left[\boldsymbol{u}(\cdot, \sigma)-\boldsymbol{u}_{\delta^{\prime}}(\cdot, \sigma)\right]\right\|_{L^{2}(\mathbb{K})} \rightarrow 0$ as $\delta^{\prime} \rightarrow 0$. This gives (27), as claimed.

In particular, we obtained (14). This, in turn, gives (15) using well known estimates of the heat operator, or, alternatively, by applying to (14) the direct method introduced in [28] to derive upper estimates in the spaces $\dot{H}^{m}\left(\mathbb{R}^{n}\right)$. This completes the proof of Theorem 1.

\section{Concluding Remarks}

The main results of this note (namely, Theorems 1 and 2) show the somewhat surprising fact that the difference of any two Stokes approximations to an arbitrarily given Leray solution of the Navier-Stokes system (1), (2) will always decay as $t \rightarrow \infty$ at least as fast as the fastest decaying Leray flows in general, no matter how slow the particular Leray solution at hand might be decaying. This is an interesting theoretical finding about Stokes flows in $\mathbb{R}^{n}$, which are important approximations for Navier-Stokes flows. On the more practical side, it sheds some additional light on the quality of these approximations. For example, it shows that M. Wiegner's estimates ([20], THeorem (c), p. 305) on the large time size of the error $\left\|\boldsymbol{u}(\cdot, t)-e^{v \Delta t} \boldsymbol{u}_{0}\right\|_{L^{2}\left(\mathbb{R}^{n}\right)}$ apply more generally to the error of any Stokes approximation of $\boldsymbol{u}(\cdot, t)$, and so forth.

Author Contributions: Conceptualization, P.R.Z.; investigation, J.C.R., J.P.Z. and P.R.Z.; writingoriginal draft preparation, P.R.Z.; writing—review and editing, J.C.R. and J.P.Z. All authors have read and agreed to the published version of the manuscript.

Funding: This research received no external funding.

Conflicts of Interest: The authors declare no conflict of interest. 


\section{References}

1. Leray, J. Essai sur le mouvement d'un fluide visqueux emplissant l'espace. Acta Math. 1934, 63, 193-248. [CrossRef]

2. Kreiss, H.-O.; Lorenz, J. Initial-Boundary Value Problems and the Navier-Stokes Equations; Academic Press: New York, NY, USA, 1989.

3. Ladyzhenskaya, O.A. The Mathematical Theory of Viscous Incompressible Flows, 2nd ed.; Gordon and Breach: New York, NY, USA, 1969.

4. Robinson, J.C.; Rodrigo, J.L.; Sadowski, W. The Three-Dimensional Navier-Stokes Equations; Cambridge University Press: Cambridge, UK, 2016.

5. Sohr, H. The Navier-Stokes Equations; Birkhäuser: Basel, Switzerland, 2001.

6. Temam, R. Navier-Stokes Equations: Theory and Numerical Analysis; AMS/Chelsea: Providence, RI, USA, 1984.

7. Silva, P.B.E.; Zingano, J.P.; Zingano, P.R. A note on the regularity time of Leray solutions to the Navier-Stokes equations. J. Math. Fluid Mech. 2019, 21, 8. [CrossRef]

8. Brandolese, L.; Schonbek, M.E. Large time behavior of the Navier-Stokes flow. In Handbook of Mathematical Analysis in Mechanics of Viscous Fluids, Part I; Giga, Y., Novotny, A., Eds.; Springer: New York, NY, USA, 2018.

9. Galdi, G.P. An introduction to the Navier-Stokes initial-boundary problem. In Fundamental Directions in Mathematical Fluid Dynamics; Galdi, G.P., Heywood, J.G., Rannacher, R., Eds.; Birkhauser: Basel, Switzerland, 2000; pp. 1-70.

10. Lorenz, J.; Zingano, P.R. The Navier-Stokes equations for incompressible flows: Solution properties at potential blow-up times. Bol. Soc. Paran. Mat. 2015, 35, 127-158.

11. Niche, C.J.; Schonbek, M.E. Decay characterization of solutions to dissipative equations. J. Lond. Math. Soc. 2015, 91, 573-595. [CrossRef]

12. Kajikiya, R.; Miyakawa, T. On the $L^{2}$ decay of weak solutions of the Navier-Stokes equations in $\mathbb{R}^{n}$. Math. Z. 1986, 192, 135-148. [CrossRef]

13. Kato, T. Strong $L^{p}$ - solutions of the Navier-Stokes equations in $\mathbb{R}^{m}$, with applications to weak solutions. Math. Z. 1984, 187, 471-480. [CrossRef]

14. Kreiss, H.-O.; Hagstrom, T.; Lorenz, J.; Zingano, P.R. Decay in time of incompressible flows. J. Math. Fluid Mech. 2003, 5, 231-244. [CrossRef]

15. Masuda, K. Weak solutions of the Navier-Stokes equations. Tôhoku Math. J. 1984, 36, 623-646. [CrossRef]

16. Oliver, M.; Titi, E.S. Remark on the rate of decay of higher order derivatives for solutions to the Navier-Stokes equations in $\mathbb{R}^{n}$. J. Funct. Anal. 2000, 172, 1-18. [CrossRef]

17. Schonbek, M.E. Large time behaviour of solutions of the Navier-Stokes equations. Commun. Partial Differ. Equ. 1986, 11, 733-763. [CrossRef]

18. Schonbek, M.E. Large time behaviour of solutions to the Navier-Stokes equations in $H^{m}$ spaces. Commun. Partial Differ. Equ. 1995, 20, 103-117. [CrossRef]

19. Schonbek, M.E.; Wiegner, M. On the decay of higher-order norms of the solutions of Navier-Stokes equations. Proc. R. Soc. Edinb. Sect. A 1996, 126, 677-685. [CrossRef]

20. Wiegner, M. Decay results for weak solutions of the Navier-Stokes equations on $\mathbb{R}^{n}$. J. Lond. Math. Soc. 1987, 35, 303-313. [CrossRef]

21. Zhou, Y. A remark on the decay of solutions to the 3-D Navier-Stokes equations. Math. Methods Appl. Sci. 2007, 30, 1223-1229. [CrossRef]

22. Gallay, T.; Wayne, C.E. Long-time asymptotics of the Navier-Stokes and vorticity equations on $\mathbb{R}^{3}$. Philos. Trans. R. Soc. Lond. 2002, 360, 2155-2188. [CrossRef] [PubMed]

23. Miyakawa, T.; Schonbek, M.E. On optimal decay rates for weak solutions to the Navier-Stokes equations in $\mathbb{R}^{n}$. Math. Bohem. 2001, 126, 443-455. [CrossRef]

24. Brandolese, L. Space-time decay of Navier-Stokes flows invariant under rotations. Math. Ann. 2004, 329, 685-706. [CrossRef]

25. Gallay, T.; Wayne, C.E. Invariant manifolds and the long-time asymptotics of the Navier-Stokes and vorticity equations on $\mathbb{R}^{2}$. Arch. Rat. Mech. Anal. 2002, 163, 209-258. [CrossRef]

26. Rigelo, J.C.; Schütz, L.; Zingano, J.P.; Zingano, P.R. Leray's problem for the Navier-Stokes equations revisited. C. R. Math. 2016, 354, 503-509. [CrossRef]

27. Schütz, L.; Zingano, J.P.; Zingano, P.R. On the supnorm form of Leray's problem for the incompressible Navier-Stokes equations. J. Math. Phys. 2015, 56, 071504. [CrossRef]

28. Hagstrom, T.; Lorenz, J.; Zingano, J.P.; Zingano, P.R. On two new inequalities for Leray solutions of the Navier-Stokes equations in $\mathbb{R}^{n}$. J. Math. Anal. Appl. 2020, 483, 123601. [CrossRef] 